\title{
Combined Electron Cyclotron Emission And Heating For The Suppression Of Magnetic Islands In Fusion Plasmas
}

\author{
H. van den Brand*, M.R. de Baar, B.A. Hennen, J.W. Oosterbeek \\ FOM Institute DIFFER - Dutch Institute for Fundamental Energy Research \\ Control Systems Technology Group, Eindhoven University of Technology, Eindhoven, the \\ Netherlands \\ E-mail: h.vandenbrandediffer.nl
}

\section{W.A. Bongers, E. Westerhof}

FOM Institute DIFFER - Dutch Institute for Fundamental Energy Research, Eindhoven, the Netherlands

\section{W. Kasparek}

Institute of Interfacial Process Engineering and Plasma Technology, University of Stuttgart, Stuttgart, Germany

\section{N. Doelman, W. Klop}

Department of OptoMechatronics, TNO Technical Sciences, Delft, the Netherlands and Leiden Observatory, University of Leiden, The Netherlands

\section{Giannone, J.K. Stober, F. Monaco, H. Schütz, D. Wagner and the ASDEX Upgrade Team \\ Max-Planck-Institut für Plasmaphysik, Garching, Germany}

\begin{abstract}
In a tokamak, magnetic islands need to be suppressed. Suppression is possible with electron cyclotron heating $(\mathrm{ECH})$, which can be positioned using electron cyclotron emission (ECE) temperature measurements. A set-up to detect ECE in the line of sight of ECH has been built for the TEXTOR tokamak and the ASDEX Upgrade tokamak. In TEXTOR, a dielectric filter was used to separate low power ECE from high power ECH and successful aiming of ECH and suppression of a magnetic island could be demonstrated. At ASDEX Upgrade, a fast directional switch (FADIS) was used to separate ECE from ECH. Measurements of magnetic islands at ASDEX Upgrade are presented. The applicability of these measurements for magnetic island detection is discussed.
\end{abstract}

First EPS Conference on Plasma Diagnostics - 1st ECPD,

14-17 April 2015

Villa Mondragone, Frascati (Rome) Italy

\footnotetext{
* Speaker.
} 


\section{Introduction}

Nuclear fusion is being developed as an energy source for future generations.[1] Fusion of the hydrogen isotopes deuterium and tritium into helium releases $17.6 \mathrm{MeV}$ and is energetically the easiest accessible fusion reaction. The optimal temperature for this reaction is $20 \mathrm{keV}$.[1] The best performing fusion plasmas to date have been achieved in a set-up design known as the tokamak, shown in figure 1. In a toroidally shaped vacuum vessel, a plasma is confined by both an external magnetic field in toroidal direction (light blue arrow) and a magnetic field in the poloidal direction (green arrows) due to the induced plasma current (green arrow in plasma). The resulting magnetic field spirals helically around the toroidal axis (purple line). In this case, the resultant magnetic field lines lie on a set of nested toroidal surfaces, called (magnetic) flux surfaces. Temperature is constant on the flux surfaces due to fast transport on these surfaces.

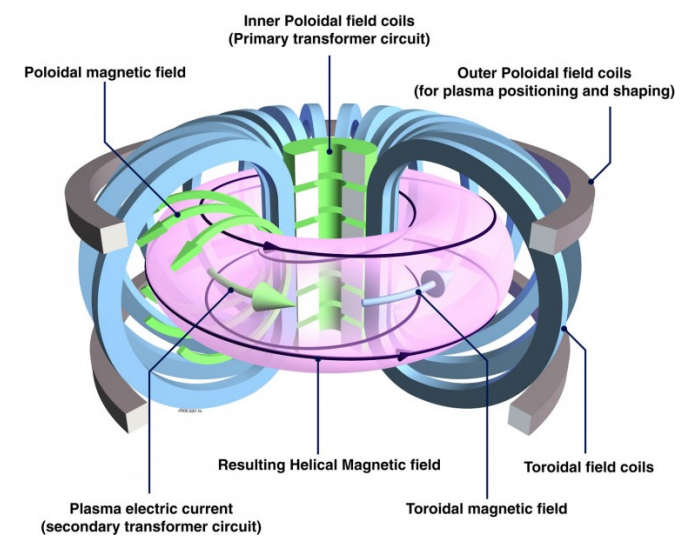

Figure 1: Tokamak principle. A poloidal field coil induces a toroidal current in the plasma and hence the poloidal magnetic field (green arrows). Toroidal field coils induce a toroidal magnetic field. The poloidal and toroidal fields result in helical magnetic field lines (purple).

Under reactor relevant conditions, slight perturbations of the current distribution can lead to the deterioration of the magnetic flux surfaces. This triggers magnetic instabilities like magnetic islands which result in an increased thermal transport from core to edge, thereby decreasing the produced fusion power and jeopardizing the stability of the entire plasma. Therefore, magnetic island control is needed.

This paper is about detection and control of magnetic islands using inline electron cyclotron emission (inline ECE). In the next section magnetic islands are presented. Important aspects for magnetic island control are presented in section 3. The idea behind inline ECE is discussed in section 4. Results and progress from the TEXTOR tokamak and the ASDEX Upgrade tokamak are presented in sections 5 and 6 . This is followed by the conclusions.

\section{Magnetic islands}

On each flux surface in the tokamak plasma, the twist of the magnetic field lines, the safety factor $q$, is a weighted ratio of the poloidal and toroidal magnetic field components. At flux surfaces with rational values $q=\frac{m}{n}$, where $m$ and $n$ are the poloidal and toroidal mode numbers, the field lines close after only $m$ toroidal turns and $n$ poloidal turns. Especially, the surfaces were $q=\frac{2}{1}$, $q=\frac{3}{2}$, and $q=\frac{4}{3}$ are known to be unstable to perturbations of the same helicity (same $m$ and $n$ ). Such perturbations result in magnetic islands that are characterized by their island width $w$.[2] For 
a specific magnetic configuration the fusion plasma is described by its safety factor profile $q(\rho)$ with $\rho$ a radial coordinate (with respect to the plasma core) which is 0 at the core and 1 at the edge. At the radial location $\rho_{m, n}$ the safety factor is equal to $q\left(\rho_{m, n}\right)=\frac{m}{n}$ and a magnetic island could be expected. Furthermore, the plasma rotates and this also results in a rotation of the magnetic island with frequency $\omega$. All these aspects are shown in figure 3. The island width $w$ is given in terms of the normalized radius coordinate $\rho$. There are numerous perturbations (e.g. sawtooth, fishbones, ELMs) that can induce a small magnetic island on a surface with rational $q$. [2] These small islands result in a self-enforcing decrease of the current which drives the growth of the island.

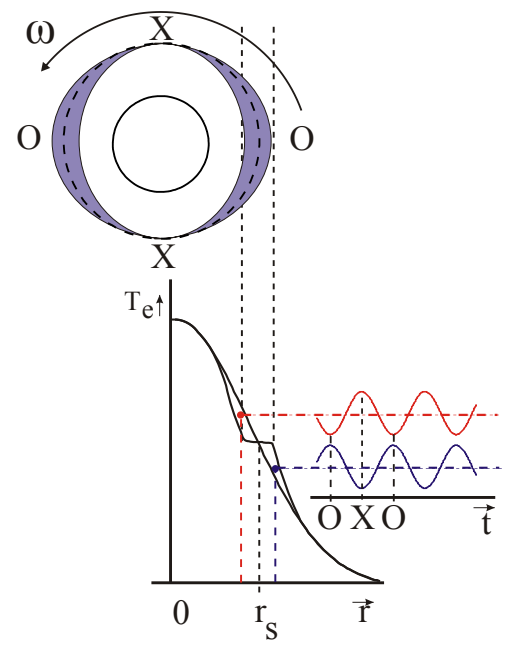

Figure 2: Schematic drawing of the temperature profile perturbation due to the magnetic island. The radial location of the magnetic island is indicated by $r_{s}\left(\rho_{m, n}\right)$. A magnetic island is shown, rotating in a poloidal projection. The temperature measured on opposite sides of the resonant flux surface shows an oscillation at the island rotation frequency $\omega$ that is in anti-phase. The temperature at $r<r_{s}$ is always higher than the temperature at $r>r_{s}$. The difference is minimal for the O-point and maximal for the $\mathrm{X}$-point.

A magnetic island forms its own set of magnetic flux surfaces around its magnetic axis, the O-point. In terms of $\rho$ of the original plasma, the separatix, the flux surface that insulates the island from the bulk plasma, connects the position $\rho_{m, n}+w / 2$ with the position $\rho_{m, n}-w / 2$ leading to a much faster radial transport. This leads to a constant temperature on the separatrix and, in the absence of heat sources in the island, to a flattened temperature in the magnetic island. The temperature perturbation as a function of $\rho$ is considered at fixed poloidal and toroidal angle. When a larger part of the island is in view, this results in a larger region in which a flattened temperature is measured. Conversely, if only the smallest part of the island is in view, the Xpoint, no flattening of the temperature is observed. The temperature difference with respect to the average temperature shows an antiphase in oscillation when comparing two measurement points on opposite sides of $\rho_{m, n}$. Furthermore, the island also perturbs the shape of the flux surfaces outside the island. Figure 2 shows these temperature fluctuations that allow for the determination of $\rho_{m, n}$. With external magnetic coils it is also possible to pick up rotating magnetic fields that correspond to selected $m$ and $n$ mode numbers.

A large magnetic island reduces the core temperature by increasing the thermal transport. Large islands can also cause disruptions, a violent event in which the plasma is suddenly lost. Both are unwanted and therefore require suppression of magnetic islands. 


\section{Magnetic island control}

Magnetic islands need to be suppressed in real-time during a plasma discharge. This is achieved by localised current drive within the island by means of electron cyclotron heating (ECH).[3] The electron cyclotron (EC) frequency depends on the magnetic field strength which varies with the major radius $R$ (distance to the main axis of the tokamak). Radiation at a frequency $f$ that matches the EC frequency at radius $R$ is absorbed at that location and locally heats the plasma. In practice, $\mathrm{ECH}$ is applied with a fixed radiation frequency and a mirror with variable angle $\alpha$ is used to position heating at a specific flux surface. This is shown with the red lines in figure 3. ECH needs to be applied on the radial position of the island $\rho_{m, n}$, such that either the resistivity is decreased and a current is driven or the deposited power can also directly drive a non-inductive current.

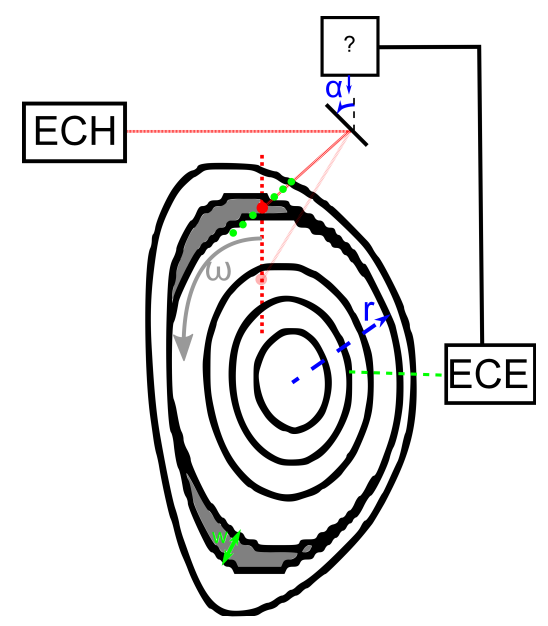

Figure 3: Crosssection of a tokamak plasma with a magnetic island and ECH and ECE. The black lines indicate the flux surfaces and the boundary of the magnetic island. The island region is colored gray. The island is located at radius $r\left(\rho_{m, n}\right)$, has an extent $w$ (expressed in normalized radius $\rho$ ) and rotates with a frequency $\omega$. ECH is positioned using a steerable mirror, by changing angle $\alpha$. A fixed frequency of ECH can be positioned along the dashed red vertical line. ECE is measuremed along a horizontal line of sight that is indicated in green. Several channels collect radiation from different locations on this line. Based on the ECE measurement ECH should be applied inside the island.

The position $\rho_{m, n}$ can be determined from a temperature measurement through the island. As explained in section 2, the antiphase measured in temperature fluctuations indicates the presence of a magnetic island in between. Electron cyclotron emission (ECE) can be used to locally measure the temperature.[4] The plasma emits this radiation at a frequency which depends on the magnetic field and therefore on the major radius. The intensity of this radiation is proportional to the local temperature (under a condition that is met in most tokamak plasmas except for the edge [4]). Typically ECE is measured along a horizontal line crossing the magnetic axis of the plasma, also shown in figure 3.

The measurements done with ECE give the position of the magnetic island along the line-ofsight of the ECE diagnostic. At ASDEX Upgrade, the phase of all ECE channels is determined relative to a combination of magnetic signals corresponding to several chosen mode number $m$ and $n$. A phase difference of $180^{\circ}$ should be observed and the fluctuation amplitude should have maxima on opposite sides of this phase difference.[5] However, the measured position needs to 
be translated to $\rho_{m, n}$ in terms of the minor radius. This requires the magnetic configuration of the plasma to be evaluated during the plasma discharge. A real-time equilibrium provides this data for ASDEX Upgrade discharges.[6] To accurately position ECH, not only the location of the island $\rho_{m, n}$ but also the density needs to be known to take refraction of the ECH beam into account. A real-time version of the TORBEAM code provides the deposition position of ECH well within $3 \%$ of the actual position.[7] On the ASDEX Upgrade tokamak, magnetic islands have been controlled using mode detection and deposition control.[8][9]

\section{Inline ECE}

Westerhof et al proposed an alternative control scheme.[10] ECE can be collected via the same mirror that is used to position ECH. ECE is thus measured in the line of ECH; inline ECE. This means that the determination of the island position is done in the same coordinate system as is used for applying ECH. Therefore, only a real-time detection algorithm is required and the number of required measurements is also reduced. If the inline ECE channels cover all (or most) of the plasma, they could localize an island regardless of the specific mirror setting. Contrarily, if the coverage of the channels is limited a real-time equilibrium and/or additional measurements are needed to position inline ECE such that it views the magnetic island. In both situations feedback, based primarily on inline ECE, is used to move the mirror when it is close to the magnetic island to put $\mathrm{ECH}$ inside the island.

However, there is a big challenge in implementing such a control scheme. ECH uses radiation in the order of $1 \mathrm{MW}$, while ECE measurements typically detect in the order of $\mathrm{nW}$. The measurement equipment for ECE is usually damaged by radiation in excess of $1 \mathrm{~mW}$. This means that the $\mathrm{ECH}$ radiation has to be suppressed at least 9 orders of magnitude. However, the frequencies at which ECE is detected should still be close to the ECH frequency to ensure accurate positioning.

A typical inline ECE set-up therefore consists of a number of parts. First of all a source for $\mathrm{ECH}$ radiation is needed. For tokamaks the gyrotron is the source of choice.[11] A path from this source to the mirror is required. Components are needed to separate ECE from the ECH radiation and finally filters are needed to suppress the ECH frequency to an acceptable level. In the next two sections two set-ups for inline ECE are discussed.

\section{Inline ECE at TEXTOR}

At the TEXTOR tokamak, ECH at $140 \mathrm{GHz}$ was used.[12] This radiation was transported using a quasi-optical transmission line. A six channel radiometer was used for the detection of ECE. This radiometer measures around $132.5 \mathrm{GHz}, 135.5 \mathrm{GHz}, 138.5 \mathrm{GHz}, 141.5 \mathrm{GHz}, 144.5 \mathrm{GHz}$, and 147.5 GHz, covering most of the plasma. Two dielectric quartz plates, placed at a fixed angle, were placed in the transmission line and were used to separate the ECE frequencies from the $140 \mathrm{GHz}$ $\mathrm{ECH}$ frequency. The two plates resulted in $60 \mathrm{~dB}$ of power suppression of the $\mathrm{ECH}$ radiation and a notch filter added another $80 \mathrm{~dB}$ of suppression. The radiometer data was processed and the results were used to apply feedback to the angle of the steerable mirror and feedback to the gyrotron power. An overview of the system is shown in figure 4.

In the TEXTOR tokamak a magnetic island was induced using the dynamic ergodic divertor (DED) coils.[13] Figure 5 shows results from TEXTOR discharge 110208.[14] In this discharge, a magnetic island was induced (amplitude depicted in blue) using the DED coils, shown in black in the top graph. The temperature oscillations due to the magnetic island were detected with the radiometer (not shown in the figure). The cross correlation of the six channels was evaluated and based on this cross correlation the expected island position was determined in terms of the ECE frequency shown in black in the bottom graph. At $2.0 \mathrm{~s}$ feedback on the island position started. As 


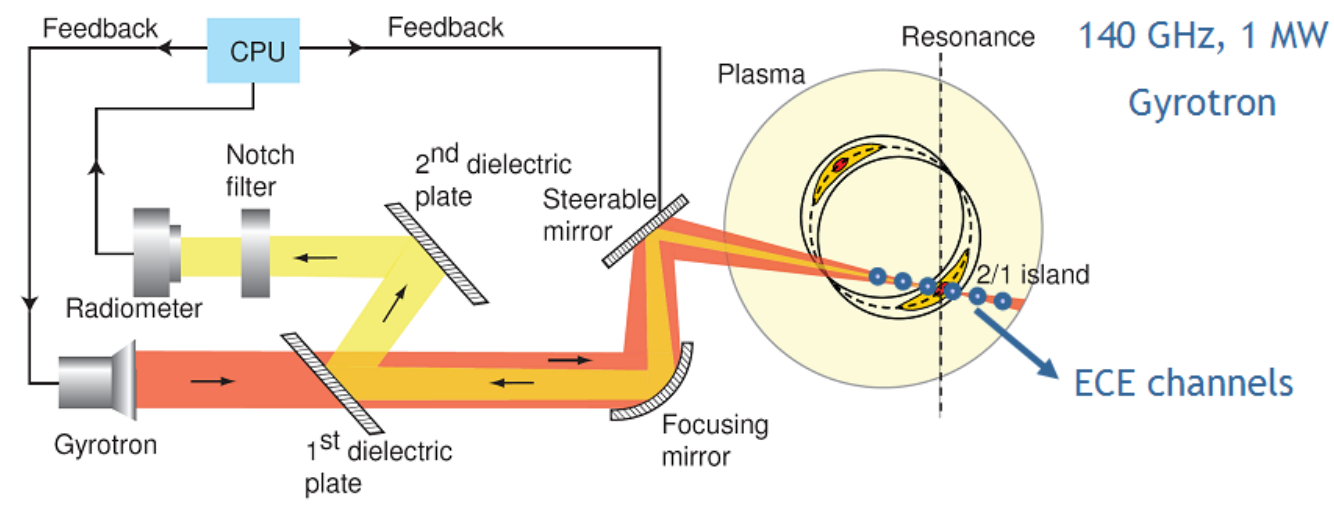

Figure 4: Schematic overview of the TEXTOR inline ECE set-up (adapted from [10]). On the right a plasma with a magnetic island is shown. A steerable mirror was used to deposit $\mathrm{ECH}$ radiation (in red) that was produced by the gyrotron. ECE radiation was collected in six channels (emission locations are indicated by blue dots). ECE was collected by the steerable mirror and was separated from the ECH radiation using two dielectric plates. A notch filter ensured sufficient suppression of ECH radiation and the radiometer measured the ECE. Measured ECE was processed (indicated as CPU block) and this provided feedback for the mirror position and the gyrotron control.

a result the steerable mirror moved (launcher elevation angle in black in the middle graph). The bottom graph shows that the mirror moved in such a way that the ECH frequency overlapped with the ECE frequency at which the island was detected. At $2.3 \mathrm{~s}$, ECH started as is shown in red in the top graph. As a result the island width decreases (blue line in top graph). From $2.5 \mathrm{~s}$ to $4.0 \mathrm{~s}$, the plasma configuration was slowly changed, as indicated by the green line in the middle graph that depicts the toroidal magnetic field. In the middle graph, it is shown that the mirror moved in response. The width of the magnetic island, shown in blue in the top graph, shows a constant island width which also confirmed that the ECH was consistently within the magnetic island.

The discharge showed that inline ECE on TEXTOR was possible. The launcher mirror was aimed based on the measured position, without incorporating additional measurements. ECH clearly reduced the island width during this controlled period. The set-up as used in TEXTOR is however not applicable for other tokamaks. Most other tokamaks use waveguides to guide ECH to the tokamak, instead of letting the ECH radiation pass through air. Furthermore, the dielectric plates heated during the experiments and as such limited the total time that ECH could be used to $3 \mathrm{~s}$.

\section{Inline ECE at ASDEX Upgrade}

At the ASDEX Upgrade tokamak, eight gyrotrons can produce $140 \mathrm{GHz}$ radiation, which is transported to the tokamak using waveguides. The fast directional switch (FADIS) is used to separate ECE from ECH radiation.[15] The use of waveguides, the possbility to do continuous wave heating through FADIS, and the H-mode plasmas result in a more reactor-relevant environment for inline ECE tests. Additional suppression of ECH radiation is achieved using a Mach-Zehnder interferometer and a Bragg reflector notch filter.[16][17] This combination of FADIS and the MachZehnder interferometer replaces the two dielectric plates in the sketch of the system given in figure 4. The same six channel radiometer, that was used for the TEXTOR experiments, is used for the detection of ECE. For typical ASDEX Upgrade conditions the ECH beam is aimed tangential to the flux surface of the magnetic island, which limits the radial coverage of the plasma with the 

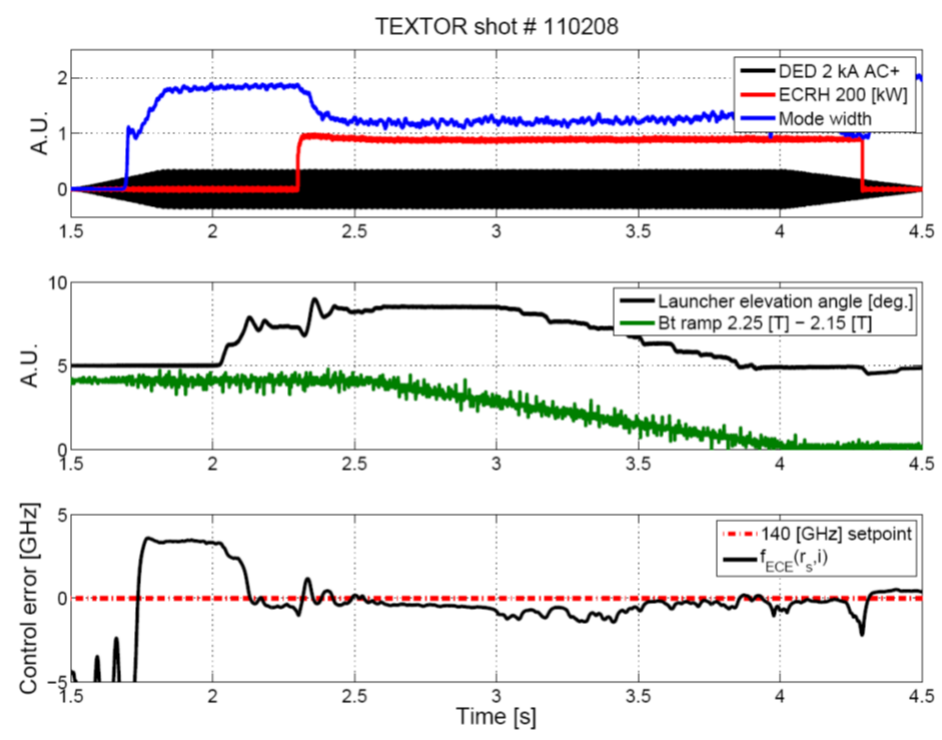

Figure 5: Part of TEXTOR discharge 110208.[14] The top graph shows the current in the DED coils in black, the island or mode width in blue and the ECH power in red. The middle graph shows the mirror angle (launcher elevation angle) in black and the toroidal magnetic field in green. The change of the toroidal magnetic field resulted in a change of the island position. The detected island position is shown in black in the bottom graph. This was based on the measurements with the six inline ECE channels (not shown). The detected position of the island is shown in terms of the ECE frequency and is compared with the $140 \mathrm{GHz}$ ECH frequency of which the difference was minimized by the controller.

six channels. In the presented results ECH is positioned based on the real-time equilibrium and real-time TORBEAM (see sect.3). Feedback based on inline ECE measurements would be done after a pre-positioning using the real-time ASDEX Upgrade control system.

A measurement of the six inline ECE channels is compared with one of the standard ECE channels. The measured intensity with the six inline ECE channels corresponds with the temperature measured in the same discharge using the standard ECE diagnostic. Therefore, it can be concluded that inline ECE presents an (uncalibrated) temperature measurement.

During ECH, several inline ECE channels show spikes as is depicted in figure 6. In this discharge, spikes are observed from $4.0 \mathrm{~s}$ to $5.5 \mathrm{~s}$, i.e. during the time that $\mathrm{ECH}$ is applied via the same sightline using FADIS. Similar spikes were observed in cases were ECE and ECH had a different sightline. All channels show some disturbances, but channels 3 and 6 show the most spikes. Box 7 shows soft X-ray measurements of which the intensity is a line-integrated measurement of the temperature.[18] The last box shows a current measurement in the divertor region in blue and an indication of edge localized modes (ELMs).[19] The spikes on the inline ECE channels occur together with spikes on the soft X-ray channels. The spikes on the soft X-ray channels result from ELMs. ELMs cause a particle exhaust which results in a current in the divertor region. The ELM detection algorithm also indicates the presence of ELMs. Therefore, the spikes on the inline ECE channels are closely related to ELMs. In TEXTOR discharges spikes in the microwave range during magnetic islands were measured and believed to be caused by the parametric decay instability.[20][21] The mechanism for the spikes at ASDEX Upgrade has not yet been understood, but a similar mechanism is expected.[22] In between the spikes there are still time intervals of roughly six times the ELM duration and therefore it would be possible to detect islands during this 
spike-free interval.

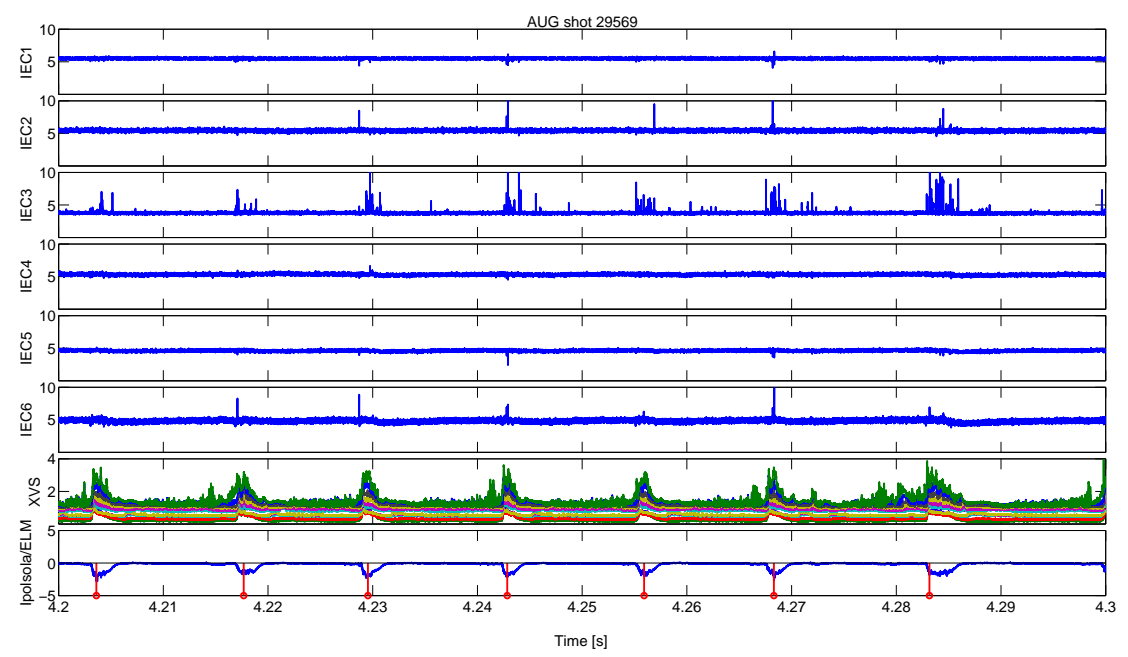

Figure 6: Zoom at the range 4.2-4.3 s of ASDEX Upgrade discharge 29569 in which an H-mode plasma was created. The six inline ECE channels are shown in the top 6 boxes. Box 7 shows soft $\mathrm{X}$-ray measurements along several sight lines in the divertor region (S2L1 from XVS). The last box shows current in the divertor in blue (Ipolsola from MAC) and the time at which edge localized modes (ELMs) are at a maximum as determined from an automatic detection algorithm. ECH is on during the entire range.

In several discharges, a magnetic island was induced. Figure 7 shows a Fourier transform of channel 5 during discharge 29573. At $2.0 \mathrm{~s}$, a magnetic island is induced. The rotation of the magnetic island clearly results in a frequency component in the inline ECE measurement. This is due to the periodic perturbation of the temperature, which is detected with inline ECE. Due to changes in the plasma the island frequency changes over time. A signal-to-noise ratio of less than 10 is observed. In the other channels the signal-to-noise ratio is lower and for channel 3 the island is only visible by comparison with other channels. Compared to TEXTOR, the noise is higher due to the higher temperature and the signal is lower due to a smaller magnetic island width. This low signal-to-noise ratio is also observed in conventional ECE measurements on ASDEX Upgrade. Based on the low signal-to-noise ratio in some channels, a refurbishment of the radiometer has been performed of which experimental results are due in the 2015/2016 ASDEX Upgrade campaign.

At ASDEX Upgrade, a dual lock-in technique significantly increases the detectability of magnetic islands in ECE signals.[5] The dual lock-in technique is applied to compare inline ECE signals with a magnetic coil signal (i.e. coil B31-13 from MHA). First, the phase of the island, as measured with the magnetic coil, is extracted using an adaptive unscented Kalman filter and this phase signal is interpolated to the time instances at which inline ECE is sampled.[23] Second, the dual lock-in technique is applied for each inline ECE to determine the correlation and the phase shift relative to the magnetic coil signal. In figure 8, six inline ECE signals from discharge 29573 are plotted around $3.0 \mathrm{~s}$ in blue. The magnetic coil signal is plotted in red where for each channel the different phase shift is taken into account and an amplitude and offset comparable to the inline ECE signal is determined. Inline ECE channels 1 and 5 are correlated for $50 \%$ with the magnetic coil signal, which is also visible in the figure. In between channel 1 and channel 5 a phase shift of $193^{\circ}$ is observed. For other channels the correlation is much less, which also results in a lack of a visible correlation in the figure. 


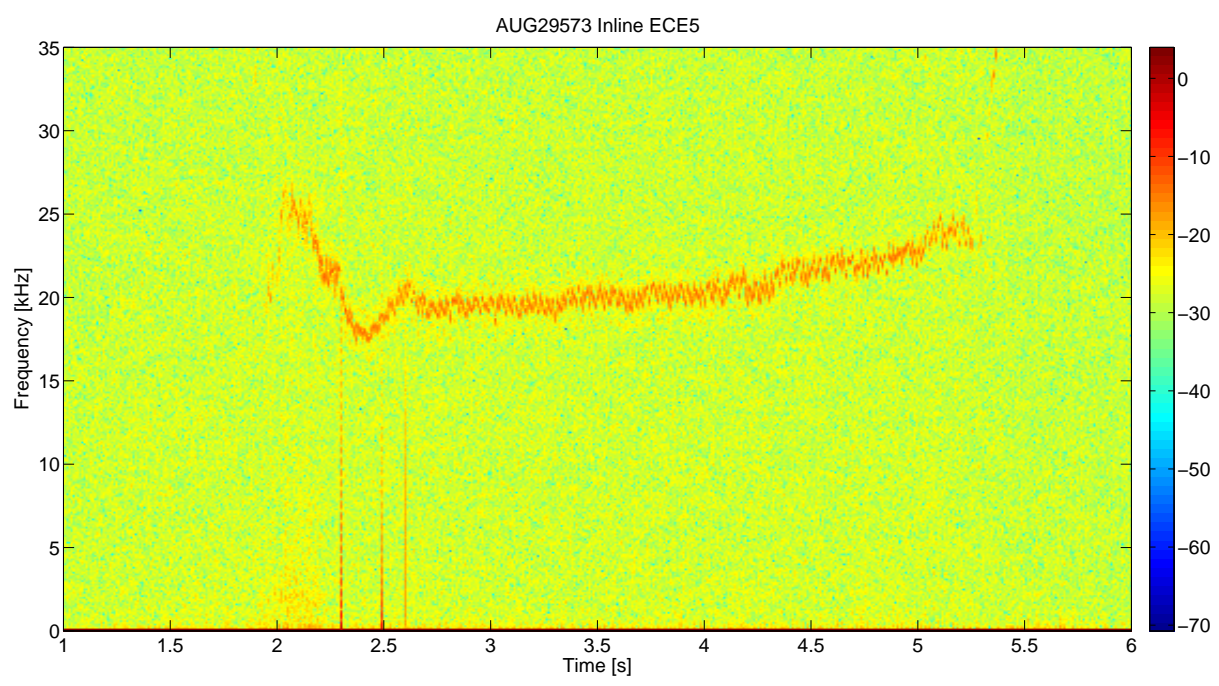

Figure 7: Fourier transform of inline ECE channel 5 for AUG discharge 29573. The amplitude of the measured voltage is calculated over periods of $10 \mathrm{~ms}$ and indicated with different colors. The scale on the right indicates the amplitude of the signals in $\mathrm{dB}$.

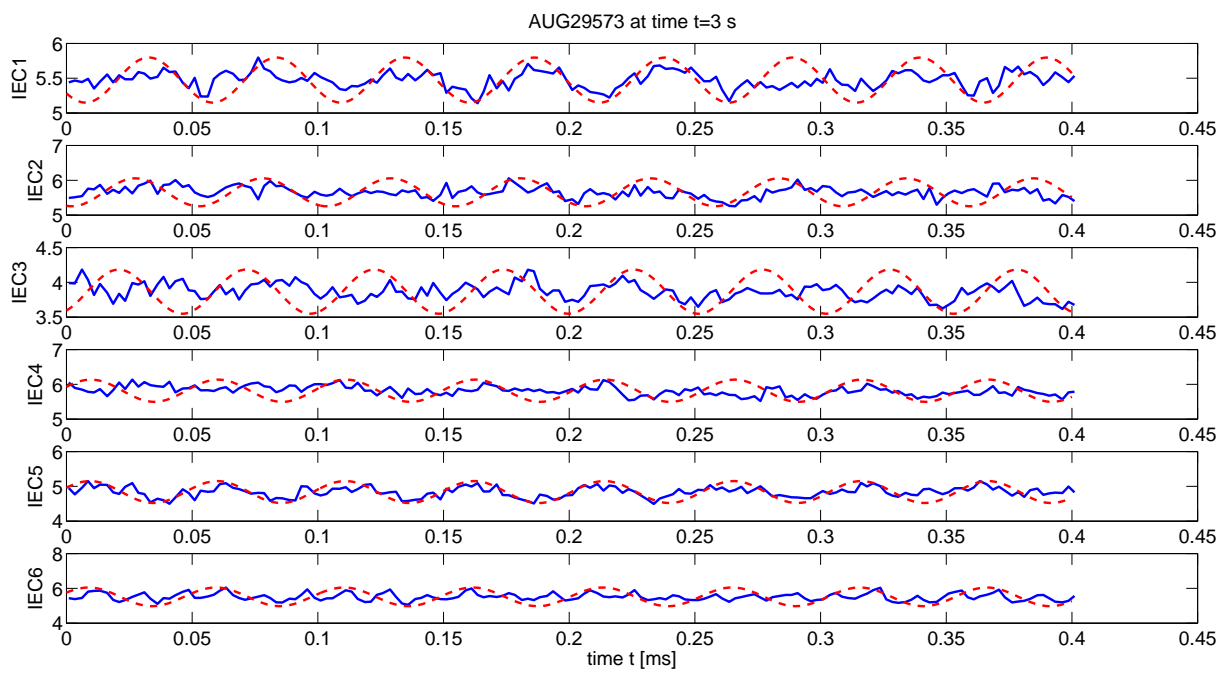

Figure 8: Raw data signals from inline ECE (blue) in discharge 29573 near $\mathrm{t}=3.0 \mathrm{~s}$. The signals are compared with a magnetic coil signal (B31-13 of MHA). For each inline ECE channel, a phase difference (determined using the dual lock-in technique) is applied to the magnetic coil signal (reddashed) and the amplitude is matched to fit the inline ECE signal.

\section{Conclusions}

In this paper, inline ECE has been presented as a means of combining ECE detection and ECH suppression for magnetic islands. The need for magnetic island suppression and the key characteristics for its detection have been introduced. The results with inline ECE at the TEXTOR tokamak 
have been highlighted and the problems for a reactor-relevant implementation are discussed.

The inline ECE set-up at ASDEX Upgrade is presented, which is a more reactor-relevant testcase and allows comparison with the ASDEX Upgrade control scheme. Measurements with inline ECE are compared with standard ECE measurements and are in agreement. Inline ECE showed spikes when ECH is applied and these spikes are most likely related to ELMs. Detection is expected to be possible in between the spikes. Inline ECE measurements showed that the magnetic island is detected. These measurements relied on launcher positioning based on a real-time equilibrium and ray tracing. With a low signal-to-noise ratio of 10 the frequency component could be detected. The raw signals showed the magnetic island only marginally. By comparison with magnetic signals it became clear that a fluctuating component, related to the island, was present in several channels, albeit at low signal-to-noise ratio. A radiometer upgrade has been performed and based on simulations the next measurements are expected to show the island fluctuations more clearly. Inline ECE feedback for ASDEX Upgrade is proposed on top of an initial launcher setting that could be based on standard ECE measurements and real-time calculations.

\section{References}

[1] G. McCracken et al. Fusion, The Energy of the Universe. Elsevier Academic Press, 2005.

[2] R. J. La Haye. Physics of Plasmas, 13(5):055501, May 2006.

[3] R. Prater. Physics of Plasmas, 11(5):2349-2376, 2004.

[4] M. Bornatici et al. Nuclear Fusion, 23(9):1153, September 1983.

[5] M. Reich et al. Fusion Science and Technology, 61(4):309-313, May 2012.

[6] L. Giannone et al. Fusion Engineering and Design, 88(12):3299-3311, December 2013.

[7] M. Reich et al. Real-time beam tracing for control of the deposition location of electron cyclotron waves (accepted for publication). Fusion Engineering and Design.

[8] M. Reich et al. In 37th EPS Conference on Plasma Physics, page P2.189, 2010.

[9] M. Reich et al. In 25th IAEA Fusion Energy Conference, pages PPC/P1-26, 2014.

[10] E. Westerhof et al. In EC-13 workshop on ECE/ECRH, Russia, 2004.

[11] Manfred Thumm. Plasma Physics and Controlled Fusion, 45(12A):A143, 2003.

[12] J. W. Oosterbeek et al. Review of Scientific Instruments, 79(9):093503, 2008.

[13] K.H. Finken et al. FUSION SCIENCE AND TECHNOLOGY, 47(2):87-96, February 2005.

[14] B. A. Hennen et al. Plasma Physics and Controlled Fusion, 52(10):104006, 2010.

[15] W. Kasparek et al. EPJ Web of Conferences, 87:04010, 2015.

[16] W.A. Bongers et al. EPJ Web of Conferences, 32:03006, 2012.

[17] D. Wagner et al. EPJ Web of Conferences, 87:04012, 2015.

[18] I. H. Hutchinson. Principles of Plasma Diagnostics. Cambridge University Press, 2002.

[19] H. Zohm. Plasma Physics and Controlled Fusion, 38(2):105, February 1996.

[20] E. Westerhof et al. Physical Review Letters, 103(12):125001, September 2009.

[21] Stefan Kragh Nielsen et al. Plasma Physics and Controlled Fusion, 55(11), 2013.

[22] Stefan Kragh Nielsen et al. Proceedings of the 9th International Workshop SMTW, 2014.

[23] D. P. Borgers et al. Fusion Engineering and Design, 88(11):2922-2932, November 2013. 